\title{
A Implementação da Telemedicina em Cabo Verde: Fatores Influenciadores
}

\section{Implementation of Telemedicine in Cape Verde: Influencing Factors}

\author{
Artur CORREIA $\triangle^{1,2,3}$, Vanda AZEVEDO ${ }^{3,4}$, Luís Velez LAPÃO 1 \\ Acta Med Port 2017 Apr;30(4):255-262 - https://doi.org/10.20344/amp.7578
}

\section{RESUMO}

Introdução: A telemedicina é a prestação de serviços de saúde, onde a distância é um factor crítico, utilizando tecnologias de informação e comunicação. Cabo Verde tem apostado na utilização desta ferramenta para aumentar o acesso das populações das suas ilhas a cuidados especializados. O objetivo deste artigo é estudar os factores influenciadores da implementação do Programa Nacional de Telemedicina no país, na vertente teleconsultas.

Material e Métodos: Estudo qualitativo, abrangendo o período entre 2013 e 2014. Utiliza a análise documental, entrevistas semiestruturadas e grupos focais, para a recolha de dados e a análise de conteúdo, para a respectiva análise. A população participante inclui médicos, enfermeiros e profissionais de algumas instituições ligadas à telemedicina.

Resultados: Os eixos prioritários do Programa Nacional de Telemedicina estão definidos, os núcleos e centros de referência estão operacionais, com pessoal formado e equipamentos instalados. Vários outros instrumentos de política e factores condicionantes $e$ facilitadores do programa foram identificados.

Discussão: A telemedicina está a contribuir para a diminuição das desigualdades no acesso à saúde, em Cabo Verde. Todavia, a adoção completa de um serviço com base numa nova tecnologia depende de factores condicionantes e facilitadores, e vários factores de sucesso da telemedicina, identificados na literatura, não são observados e conjuntamente com outras fraquezas constatadas, afectam o desenvolvimento integral do Programa Nacional de Telemedicina. Contudo os pontos fortes e as potencialidades apontados são oportunidades para agir.

Conclusão: Apesar dos avanços, alguns factores de sucesso da telemedicina apontados na literatura não se observam no país.

Palavras-chave: Acesso aos Serviços de Saúde; Cabo Verde; Saúde Global; Telemedicina

\section{ABSTRACT}

Introduction: Telemedicine is the provision of health services, where distance is a critical factor, using information and communication technologies. Cape Verde has bet on using this tool to increase access of the population of its islands to specialized care.

Material and Methods: Qualitative study, covering the period between 2013 and 2014. It uses document analysis, semi-structured interviews and focus groups to collect data and analysis of content for their analysis. The participant population includes doctors, nurses and professionals from some institutions related to telemedicine.

Results: The priorities of the National Telemedicine Program are set, the cores and reference centers are operational, with trained personnel and equipment installed. Several other policy instruments and conditioning factors and facilitators of the program have been identified.

Discussion: Telemedicine is contributing to the reduction of inequalities in access to health, in Cape Verde. However, the full adoption of a service based on a new technology depends on conditioning factors and facilitators, and several success factors of telemedicine, identified in the literature, are not observed and in conjunction with other existing weaknesses affect the overall development of the National Telemedicine Program. However the strengths and capabilities are highlighted opportunities to act.

Conclusion: Despite the progress, some telemedicine success factors highlighted on the literature are not seen in the country.

Keywords: Cape Verde; Global Health; Health Services Accessibility; Telemedicine

\section{INTRODUÇÃO}

Segundo a Organização Mundial de Saúde a telemedicina é "A prestação de serviços de saúde, onde a distância é um factor crítico, utilizando tecnologias de informação e comunicação para o intercâmbio de informações válidas para o diagnóstico, tratamento e prevenção de doenças e lesões, pesquisa e avaliação, e para a contínua educação dos profissionais de saúde, com o fim de promover a saúde dos indivíduos e de suas comunidades". ${ }^{1}$

A telemedicina surge assim como ferramenta para potenciar um uso mais efectivo de recursos, aproximar os cuidados de saúde ao cidadão e promover a modernização dos processos, métodos de trabalho e a mudança organizacional nas unidades de saúde., ${ }^{2,3}$ Todavia, esta ferramenta ainda não atingiu o seu potencial para ser usada em larga-escala, sobretudo porque a abordagem tem seguido uma perspetiva essencialmente tecnológica. ${ }^{4,5}$

A condição arquipelágica de Cabo Verde, sempre impôs desigualdades no acesso à saúde. Contudo, numa perspectiva de acesso universal, as evacuações médicas internas e externas e a deslocação de especialistas representam uma valiosa contribuição para a minimização dessas desigualdades, apesar do país não dispor de médicos

1. Global Health and Tropical Medicine. Instituto de Higiene e Medicina Tropical. Universidade NOVA. Lisboa. Portugal.

2. Comité de Coordenação do Combate à SIDA. Praia. Cabo Verde.

3. Ministério da Saúde de Cabo Verde. Praia. Cabo Verde.

4. Colégio de Cardiologia. Ordem dos Médicos de Cabo Verde. Praia. Cabo Verde.

$\square$ Autor correspondente: Artur Correia. arturcorreia51@hotmail.com

Recebido: 02 de março de 2016 - Aceite: 18 de novembro de 2016 | Copyright @ Ordem dos Médicos 2017 
especialistas suficientes para dar resposta à demanda (seis médicos, incluindo especialistas, por 10000 habitantes). ${ }^{6}$

De realçar ainda, que em 2012 foram registadas perto de duas mil solicitações para evacuações internas em Cabo Verde. ${ }^{7}$

Neste contexto, como estratégia para melhorar a eficácia e a eficiência da resposta, em 2012, o Governo, através do Ministério da Saúde, decidiu implementar um programa de telemedicina, interligando as estruturas de saúde dos níveis primário, secundário e terciário. ${ }^{8}$

De salientar que o sistema de saúde de Cabo Verde também discute e envia doentes para outros países (e.g. Portugal), utilizando a plataforma de telemedicina. ${ }^{9}$

O objectivo deste artigo foi estudar os factores influenciadores da implementação do Programa Nacional de Telemedicina (PNT) nos serviços de saúde públicos de Cabo Verde, dando ênfase às medidas de política, às infraestruturas, aos processos e sistemas implementados e aos resultados conseguidos.

Convém salientar que o caso de Cabo Verde, pelo seu carácter arquipelágico e com uma população relativamente pequena e poucos recursos é muito relevante em termos de saúde global.

\section{A telemedicina em Cabo Verde}

Várias iniciativas de cooperação técnica foram protagonizadas pelos hospitais centrais do país, com alguns centros hospitalares estrangeiros, com recurso a plataformas diversas de governação electrónica em saúde, proporcionando teleconsultas e telediagnósticos. ${ }^{10}$ Segundo esse autor, em 2000, foi organizado, com o apoio da Embaixada dos Estados Unidos da América, uma intervenção de telemedicina com a ilha Brava, através de uma linha ISDN $128 \mathrm{Ks}$, e do Net Meeting, para a transmissão de imagem de radiografia. Em 2007, com o apoio da cooperação espanhola, os dois hospitais centrais do país foram conectados entre si e com um centro hospitalar em Espanha. ${ }^{10}$

Em 2009, com apoio do Serviço de Cardiologia do Centro Hospitalar de Coimbra e com o patrocínio da CVTelecom e da PT Multimédia, teve início a primeira ligação entre aquele serviço e o Serviço de Cardiologia do Hospital Dr. Agostinho Neto, na Praia. ${ }^{11}$ Seguiu-se a ligação ao segundo hospital central em 2012, no Mindelo. ${ }^{12}$ Esta iniciativa, no domínio da cardiologia pediátrica, perdura até hoje, através de teleconsultas periódicas, discussão de casos e programação de evacuações de Cabo Verde para Portugal.

Em 2010, com o apoio da cooperação Indiana, foi possível a integração de Cabo Verde na Comunidade Médica Africana e da Índia, através do projeto Pan African e Network. ${ }^{13}$ Este projeto permite a Cabo Verde a apresentação e discussão de casos (store and forward) e é composto de uma plataforma e-learning.

Em 2012, o percurso da telemedicina em Cabo Verde conheceu uma viragem estratégica fundamental com a implementação de um projeto de infraestrutura de serviços de telemedicina, financiado pela Cooperação Eslovénia, em associação com a International Telemedicine Founda- tion (ITF). ${ }^{14}$ Este projeto permitiu, nas suas duas fases, a ligação entre os hospitais centrais e regionais, em conexão com as delegacias de saúde, em todas as ilhas, até finais de 2013. Os hospitais centrais desempenham o papel de centros de referência.

No âmbito desse projeto, vários profissionais e técnicos de saúde, das diversas estruturas, receberam formação, no país e no exterior, com vista à apropriação e utilização dos recursos disponibilizados pela telemedicina.

Nesta sequência, em 2013, por despacho da Ministra Adjunta e da Saúde, de 25 de novembro, ${ }^{15}$ foi aprovada a lista de membros dos núcleos de telemedicina, em todas as ilhas. Nesse mesmo ano, foi criada uma página no Facebook (cardiocenter), adstrita aos profissionais praticantes da telemedicina e colaboradores, destinada à partilha de informações e documentação e motivação de profissionais.

Em 2014, por despacho $n^{\circ} 2$ da Ministra Adjunta e da Saúde, de 17 de janeiro, foi criado, oficialmente, o Programa Nacional de Telemedicina. ${ }^{16}$ No mesmo despacho, foram definidos os eixos prioritários e os objetivos do programa. Por despacho de 31 de janeiro do mesmo ano da Ministra-adjunta e da Saúde, é nomeado o coordenador do PNT. ${ }^{17}$ Nesse mesmo ano, foi aprovado um conjunto de normas organizativas e funcionais desse programa. ${ }^{18}$

A equipa do PNT é constituída ao nível central por um diretor (médico) e um secretariado, constituído por um administrativo e um engenheiro informático que prestam apoio logístico e técnico. Ao nível das estruturas de saúde nos concelhos e ilhas, existem os núcleos de telemedicina, constituídos por um clínico geral (coordenador), um enfermeiro e um técnico de informática. Além disso, funcionam, ao nível dos hospitais centrais, os centros de telemedicina, com um coordenador (médico) e um administrativo, prestando apoio logístico e administrativo para a gestão dos pedidos de teleconsultas.

Em 2014, todos os oito núcleos existentes em todas as ilhas e os dois centros de referência, nos dois hospitais centrais, já estavam operacionais para teleconsultas, graças à instalação dos equipamentos e a consequente formação de pessoal técnico sobre o manuseamento dos equipamentos, e sobre os procedimentos das teleconsultas. ${ }^{19}$

Os clínicos gerais das delegacias de saúde, podem solicitar teleconsultas aos médicos especialistas em tempo real ou em diferido. ${ }^{18} \mathrm{O}$ sistema permite igualmente o envio de imagens de radiografia, eletrocardiogramas e ecografias. A marcação de teleconsultas é diária, existindo um calendário para as especialidades, nos dois centros de referência, nos hospitais centrais. Não existem pagamentos para teleconsultas.

A rede de telemedicina existente utiliza a plataforma comercial, financiada através de acordo de parceria, existente entre o Hospital Central da Praia/Centro Nacional de Telemedicina e a NOSI (Núcleo Operacional para a Sociedade da Informação) CVTelecom.

\section{Evolução das teleconsultas}

Conforme nos dá conta o relatório do PNT de $2013,{ }^{20}$ as 
atividades de telemedicina interna começaram somente a partir do mês de maio desse ano. As especialidades mais solicitadas foram, por ordem decrescente a neurologia e a cardiologia, seguidas de cirurgia, dermatologia e ortopedia, representando, conjuntamente, cerca de $65 \%$ das teleconsultas.

Segundo o PNT, ${ }^{19}$ o Hospital Central da Praia suportou $74 \%$ das teleconsultas produzidas em 2014 e a grande maioria das teleconsultas foi dada em 'tempo real' para os serviços de saúde das llhas.

Com a implementação dos serviços de teleconsultas em 2014, o número de evacuações médicas internas reduziu-se consideravelmente, uma vez que se tornou norma a realização prévia de uma teleconsulta, para se avaliar a necessidade ou não da evacuação. Com efeito, somente $18 \%$ de doentes sujeitos a uma teleconsulta foi evacuada, o que significa uma alta taxa de diagnósticos feitos directamente através de teleconsultas. ${ }^{19}$

\section{MATERIAL E MÉTODOS}

Trata-se de um estudo qualitativo sobre a implementação do Programa Nacional de Telemedicina, em Cabo Verde, relativo ao período 2013 e 2014, que utiliza a análise documental, entrevistas semiestruturadas e grupos focais, para a recolha de dados. A análise documental pretendeu pesquisar os documentos relativos à telemedicina, produzidos no período entre 2009 e 2014, como sejam relatórios elaborados a nível dos departamentos centrais do Ministério da Saúde, nos hospitais centrais e pelo Programa Nacional de Telemedicina, documentação diversa, despachos, normas e procedimentos relativos à implementação da telemedicina no país.

Para uma melhor perceção dos constrangimentos, dos pontos fortes e das fraquezas e ameaças à implementação do Programa Nacional de Telemedicina, realizaram-se 10 entrevistas semiestruturadas e dois grupos focais (um de seis e outro de oito elementos).

A população participante nas entrevistas (50\% homens e $50 \%$ mulheres) pertencia, na sua maioria, ao grupo etário 30 - 34 anos (Tabela 1). Era constituída por diversos profissionais atores da telemedicina, entre os quais médicos clínicos gerais e especialistas (delegados de saúde, o responsável do Programa Nacional de Telemedicina, diretores clínicos de hospitais e o próprio Diretor Nacional de Saúde). Nos grupos focais, a população participante (57\% homens e $43 \%$ mulheres) pertencia, na sua maioria, aos grupos etários 30 - 34 e 45 - 49 anos (Tabela 1). Integrou, além de médicos e enfermeiros, representantes do Instituto Nacional de Previdência Social, do Núcleo Operacional para Governação Electrónica, das universidades e da Agência Nacional de Telecomunicações.

A análise das entrevistas e dos grupos focais foi feita através da técnica de análise de conteúdo. Além disso, a partir da análise de conteúdo, foi feita uma análise SWOT (Tabela 2).

O protocolo da pesquisa estudo foi submetido ao Comité de Ética Nacional que o aprovou.

\section{RESULTADOS}

Dividiu-se a apresentação de resultados em três temas: políticas, infraestrutura e processos e sistemas.

\section{Políticas}

A análise documental mostra-nos que, além da aquisição e instalação dos equipamentos, foi necessário formar uma equipa sobre a operacionalização dos equipamentos e sobre a temática 'Telemedicina', nos diferentes núcleos de telemedicina criados, nas várias ilhas, ao nível das delegacias de saúde e dos hospitais regionais, e, também, ao nível dos centros de telemedicina nos dois hospitais centrais. Foi, ainda, necessário criar uma equipa dinamizadora ao nível da direção do Programa de Telemedicina.

A análise documental mostra-nos, ainda, que através do despacho de 25 de março de 2013 da Ministra Adjunta e da Saúde, foram nomeados os membros dos Núcleos de Telemedicina. Nesse mesmo ano, foi criada, pela direção do Programa Nacional de Telemedicina, uma página no Facebook para a partilha de informações e documentação, e motivação de profissionais de saúde. Em 2014, através do despacho $\mathrm{N}^{\circ} 2$ da Ministra Adjunta e da Saúde, de 17 de janeiro, oficializou-se o Programa Nacional de Telemedicina e definiram-se os respectivos eixos prioritários e objectivos. No mesmo ano, foi nomeado o coordenador do PNT e aprovado um conjunto de normas organizativas e funcionais. ${ }^{16,17}$ As teleconsultas iniciaram-se em 2013 com envolvimento de alguns núcleos de telemedicina e de algumas especialidades, e tiveram um incremento substancial, em 2014, chegando a todos os núcleos e envolvendo, praticamente, todas as especialidades médicas existentes nos dois hospitais centrais do país.

Tabela 1 - Distribuição dos participantes na pesquisa, por sexo, grupo etário e método de recolha de dados

\begin{tabular}{|c|c|c|c|c|c|c|c|c|c|c|c|c|}
\hline \multirow{2}{*}{$\begin{array}{l}\text { Técnicas de } \\
\text { recolha de } \\
\text { dados }\end{array}$} & \multirow[b]{2}{*}{ Sexo } & \multirow[b]{2}{*}{$20-24$} & \multirow[b]{2}{*}{$25-29$} & \multirow[b]{2}{*}{$30-34$} & \multicolumn{3}{|c|}{ Grupo Etário } & \multirow[b]{2}{*}{$50-54$} & \multirow[b]{2}{*}{$55-59$} & \multirow[b]{2}{*}{$60-64$} & \multirow[b]{2}{*}{$\geq 65$} & \multirow[b]{2}{*}{ Total } \\
\hline & & & & & $35-39$ & $40-44$ & $45-49$ & & & & & \\
\hline \multirow{2}{*}{ Entrevista } & M & 0 & 0 & 2 & 0 & 0 & 1 & 0 & 0 & 1 & 1 & 5 \\
\hline & $\mathbf{F}$ & 0 & 0 & 2 & 0 & 0 & 0 & 2 & 1 & 0 & 0 & 5 \\
\hline \multirow{3}{*}{$\begin{array}{l}\text { Grupos } \\
\text { focais }\end{array}$} & M & 0 & 0 & 2 & 0 & 0 & 3 & 0 & 1 & 1 & 1 & 8 \\
\hline & $\mathbf{F}$ & 0 & 1 & 1 & 0 & 1 & 1 & 2 & 0 & 0 & 0 & 6 \\
\hline & Total & 0 & 1 & 7 & 0 & 1 & 5 & 4 & 2 & 2 & 2 & 24 \\
\hline
\end{tabular}

M: Masculino; F: Feminino 
Tabela 2 - Análise SWOT das entrevistas e grupos focais

\section{Forças}

- Existência de nova infraestrutura técnica (equipamentos e rede) de telemedicina.

- Melhorias na abordagem aos doentes submetidos a teleconsultas, com possibilidades de um diagnóstico mais precoce.

- Melhorias na Comunicação entre os profissionais dos hospitais centrais e regionais.

- Realização de teleformações, assistência a sessões clínicas e congressos, de cariz nacional e internacional.

- O potencial de diminuição de custos (evacuações, deslocação de especialistas, formação, etc.).

- Inovação nos processos e na forma de trabalhar dos serviços.

- Acesso a melhores práticas na abordagem ao doente.

- Potencialidades para a internacionalização (assistência a teleformação, sessões clínicas, congressos, etc.), através de videoconferência.
Fraquezas

- Pouco envolvimento de alguns médicos especialistas nos hospitais e clínicos gerais nas delegacias de saúde.

- Poucas atividades de informação, educação e comunicação, junto da população e profissionais sobre a Telemedicina.

- Deficiências de articulação entre os serviços, no processo de marcação das teleconsultas.

- Insuficiências no funcionamento dos serviços de marcação de teleconsultas.

- Insuficiente formação dos novos técnicos integrados nos serviços, para a utilização dos equipamentos.

- Resistência à mudança, na introdução da telemedicina, por parte de alguns profissionais (médicos, enfermeiros), a diversos níveis.

- Desmotivação de alguns profissionais devido à carga de trabalho.

- Perceção de limitações que levam à quebra de privacidade nas teleconsultas, pela presença na sala de outros profissionais, além de médicos.

\section{Oportunidades}

- Abertura da Telemedicina ao privado, ligando instituições fora da rede pública.

- Possibilidade de desenvolvimento de Telemedicina entre Cabo Verde e países amigos.

- Potencialidades para fornecimento de teleconsultas a países amigos e para apoiar as nossas comunidades emigradas, nomeadamente em S. Tomé e Príncipe.

\section{Infraestruturas}

A análise de conteúdo das entrevistas e dos grupos focais permite destacar os seguintes aspectos da implementação da telemedicina em Cabo Verde e transcrever algumas abordagens dos participantes no estudo:

- Existência de nova infraestrutura técnica (equipamentos e rede) de telemedicina

P1: “... todas as ilhas conectadas à rede de telemedicina... $3^{a}$ fase do programa, em que vamos ter todos os centros de saúde ligados, à rede de telemedicina..."

P2: “... muitas vezes o sistema não está disponível no momento, muitas vezes conseguimos visualizar as pessoas, outras vezes nem tanto..."

- Existência de uma rede de fibra ótica a conectar as diferentes ilhas

P3: “... do ponto de vista das infra-estruturas das telecomunicações, da rede, acho que, ... estamos bem dotados, porque existe uma infra-estrutura de fibra óptica, quase espalhada por todos os concelhos, por meio terrestre, e as ilhas estão todas ligadas pela fibra óptica em anel." "Uma outra parte também é a questão da priorização
- Avarias pontuais (máximo três dias de duração) nos sistemas de comunicação.

- Problemas ligados à sustentabilidade financeira para manter actualizados os equipamentos.

- Deficiências na articulação com o Núcleo Operacional para a Governação Eletrónica do Estado (NOSI).

do tráfico da rede da telemedicina, que é um aspecto que também deve ser estudado com antecedência e calma, para que o tráfico que é proveniente de um equipamento que está ligado à rede de telemedicina possa ser priorizado."

P4: "Naturalmente que nós temos outras vantagens no país, que facilita isso, são: as redes de telecomunicação, nós podemos não estar no óptimo, mas estaremos no bom, em termos de rede de telecomunicação, que permite esta interligação, e falarei também da questão da energia eléctrica, da rede de energia eléctrica espalhada pelo país, que permite também instalar e fazer funcionar os equipamentos, já de uma forma bem descentralizada, e portanto alargar ao país completo ou quase, esse benefício."

\section{- Existência de alta taxa de cobertura de internet e da rede móvel no país}

\section{- Avarias nos sistemas de comunicação}

P5: "...muitas vezes temos algumas falhas de comunicação na transmissão, por ex.: de ligação." 
P6: "A questão dos constrangimentos que se fala mais tem a ver com o sistema. Estamos no meio de algo e de repente nos cai o sistema."

- Problemas ligados à sustentabilidade financeira para a renovação dos equipamentos

P4: "... um sistema desses implica financiamentos, investimentos pesados, porque adquirir todo o equipamento e equipar todos os centros, custa, mas não é, no meu entender, um impeditivo ... não é com certeza aquilo que mais impedirá o seu desenvolvimento."

\section{Processos e sistemas}

- Disponibilidade de técnicos e especialistas

P1: "....A falta de técnicos de informática nos diversos centros, o que dificulta, às vezes, as emissões..."

- Inovação nos processos e na forma de trabalhar dos serviços

P7: “... é uma tecnologia que veio para ficar, num quadro de reforma institucional bem mais alargado, que é de aproximação dos cuidados de saúde à população. Portanto, não sendo possível deslocar todas as tecnologias e todos os especialistas para junto do utente, exactamente, a telemedicina faz aqui esta mediação entre o utente e esses recursos..."

"Desde logo, a telemedicina aqui pode, também, trazer alguma densidade tecnológica virtual para os centros de saúde e é claro, a questão da criação de equipas multidisciplinares, portanto, tem que ter esta equipa multidisciplinar nesta retaguarda virtual que a telemedicina proporciona."

P1: "Mas isto também é uma situação interessante, as colegas que estão na Boavista se dão muito bem com as colegas da ilha do Sal. E como têm lá a medicina interna, a ginecologia e a pediatria, fazem teleconsulta entre eles, sem passar pelo hospital central. É uma dinâmica diferente e esse é o futuro também."

- Resistência à mudança de alguns profissionais (médicos, enfermeiros), a diversos níveis, na introdução da telemedicina

P7: “... alguns colegas, com mais afinidade às questões da tecnologia, fazem mais uso, mas por vezes nós sentimos alguma resistência e nós compreendemos que esta resistência muitas vezes tem a ver com essa facilidade ou não no trato, no manejo da tecnologia."

"... algumas resistências do profissional é uma resistência que é reactiva à "estranheza" do próprio utente que desconhece e que, de repente, o técnico diz ok, você vai ter a consulta com o cardiologista, mas sem ter a mão do cardiologista a tocar."

P8: “... na realidade nós sabemos que todos nós somos de alguma forma resistentes à mudança ... É um aspecto novo, nós temos que trabalhar mais, até esta tecnologia estar implementada nas nossas rotinas. Então eu acho que é preciso insistir mais no processo de empodera- mento dos profissionais de saúde ... do apropriamento do sistema ... é só a partir daí que nós vamos conseguir..."

- Pouco envolvimento ou desmotivação de alguns médicos especialistas nos hospitais e clínicos gerais nas delegacias de saúde

P8: "Eu acho que, em primeiro lugar, é preciso trabalhar mais na questão do apoderamento da telemedicina pelos profissionais de saúde, portanto, muitas vezes eu sinto na minha estrutura que muitos colegas, eu estou a falar de colegas médicos, ainda encaram a telemedicina como mais uma obrigação, uma burocracia, e não entenderam, o quão importante é a telemedicina ..."

P9: "Não há um envolvimento, ainda, de todos os médicos nas teleconsultas, há necessidade de uma sensibilização dos médicos mas também, acredito que a divulgação da telemedicina no seio dos profissionais saúde foi insuficiente."

- Poucas atividades de informação, educação e comunicação, junto da população e profissionais sobre a telemedicina

P10: "Eu acho que sensibilização dos colegas é um desafio grande, não só da neurologia mas das outras especialidades também."

P11: “...é a apropriação da telemedicina pelos seus actores, porque isso passa ainda pala formação, capacitação e sensibilização dos seus actores, porque ainda de momento se você for ver, muita gente olha a telemedicina com alguma reticência."

- A comunicação entre os profissionais, através da telemedicina

P9: "... A telemedicina, a meu ver, acabou com o isolamento do médico nas nossas ilhas."

P4: "... e em termos da presença dos colegas no outro local, e, além disso, naturalmente, rompe com a solidão que nós que estivemos já nas ilhas, sozinhos ou quase, estamos voltados para à solidão e esto é mau, sobretudo, para os profissionais, mas é mau para as pessoas."

- Insuficiências na articulação entre os serviços, no processo de marcação das teleconsultas

P12: “...porque às vezes o colega fica muito tempo a esperar o outro colega para ir fazer a telemedicina, e não só, também, às vezes algumas consultas para determinar se o paciente vai para Praia ou não, a colega que vai fazer telemedicina não tem um relatório para enviar, para comprovar que fizemos telemedicina ..."

P1: “... às vezes o médico que está com o doente e o especialista, o médico chega e fica à espera do especialista, o especialista chega e fica à espera do médico. Isto acaba por ser um constrangimento na satisfação do utente." 
- Deficiências na formação dos novos técnicos integrados nos serviços, para a utilização dos equipamentos

P8: "... a segunda questão é aprofundar na formação, portanto, eu penso que alguns colegas do hospital tiveram uma formação muito boa, mas a passagem desses conhecimentos sobre a utilização, sobre a exploração do aparelho, da tecnologia, porque na realidade as pessoas sentem-se à vontade com aquilo que conhecem e dominam, não é?"

P7: “... nos próximos tempos, tem que se continuar com as actividades de formação, mas aqui no pais, já temos um núcleo duro com competência nesta matéria, que pode, perfeitamente, realizar formações anuais, anuais mesmo, para os recém-chegados..."

- Realização de teleformações, assistência a sessões clínicas e congressos, de cariz nacional e internacional, com a consequente actualização técnica e satisfação dos profissionais

P9: “... a telemedicina vai permitir a formação contínua, a educação contínua à distância dos profissionais de saúde ..."

P4: “... irão beneficiar certamente destas novas tecnologias, permitindo o ensino à distância, tão bem quanto presencial, com ganhos naturais, em termos de tempo, de deslocações ..."

- A abordagem aos doentes submetidos a teleconsultas, com possibilidades de um diagnóstico mais precoce

P12: "... ajuda o médico a pensar, a determinar e a diagnosticar melhor o doente, e quem tem tudo a ganhar é o paciente, o médico e o sistema ..."

P1: "Todos os electrocardiogramas podem ser enviados para um dos centros e ser lido por um técnico de cardiopneumologia ... portanto com esta leitura, nós fazemos diagnósticos mais precoces, e mandam-se resultados atempadamente."

- Acesso a melhores práticas na abordagem ao doente

P9: "O médico na ilha poderá ter parecer sobre o diagnós- tico, será auxiliado na orientação clínica, será auxiliado no tratamento do doente e mesmo aquele doente que tiver necessidade de evacuação, porque é nos hospitais que nós temos concentrado as tecnologias e os cuidados de maior complexidade; ... depois do regresso à ilha, podemos efectuar o seguimento do doente ..."

"... O doente... em relação à teleconsulta pode até ter teleconsulta multidisciplinar."

\section{DISCUSSÃO}

A utilização da telemedicina está progredindo rapidamente e pode representar uma alternativa para complementar muitos programas de saúde. ${ }^{21}$ Contudo, a adoção de uma nova tecnologia ou serviço depende de vários factores e etapas. ${ }^{22} \mathrm{Na}$ primeira etapa, impõe-se a familiarização dos indivíduos, ou do organismo, com a tecnologia; na segunda é necessária a formação de uma opinião favorável ou desfavorável sobre a nova tecnologia; seguindo-se a etapa de adoção ou de rejeição da tecnologia.

A Tabela 3 mostra-nos os fatores críticos de sucesso, apontados por Kodukula e Nazvia ${ }^{23}$ para a implementação da telemedicina.

Em Cabo Verde, três desses dez factores apresentam algumas dificuldades na sua observância, nomeadamente os relativos a 'ter disponibilidade de fundos de forma sustentável', 'ter recursos humanos adequados e capacitados' e 'garantir comunicação e articulação entre as partes interessadas'. Com feito, a análise SWOT do Programa Nacional de Telemedicina, feita a partir da análise de conteúdo das entrevistas e dos grupos focais, permitiu constatar os seguintes constrangimentos: a implementação da telemedicina depende de fundos da cooperação internacional; a mobilidade dos recursos humanos dificulta o processo de implementação desta intervenção, uma vez que obriga a repetição das formações relativas ao uso dos equipamentos e aos procedimentos e condutas a ter no processo das teleconsultas e, por último, ainda existem lacunas na comunicação a articulação entre as partes interessadas.

Lange ${ }^{24}$ dá conta dos resultados de estudos levados a cabo, nos diferentes países europeus, pelo Projecto MOMENTUM, sobre os factores críticos de sucesso a serem levados em conta, na implementação da telemedicina. Esses estudos permitiram destacar 18 factores críticos de

Tabela 3 - Fatores críticos de sucesso, para a implementação da telemedicina. Adaptado de Kodukula S, Nazvia M. Evaluation of critical success factors for telemedicine implementation. Int J Computer Applications. 2011;12:29- 36. ${ }^{23}$

\begin{tabular}{ll}
\hline Factores críticos de Sucesso & Observância em Cabo Verde \\
\hline a) Estar apoiado nos Regulamentos e Políticas Governamentais; & Sim \\
b) Adotar um projeto de gestão padronizado; & Sim \\
c) Ter a aceitação do público; & Sim \\
d) Ter apoio político; & Sim \\
e) Ter a disponibilidade de infraestrutura tecnológica, incluindo hardware, software e adequada & Sim \\
largura de banda; & Não \\
f) Ter disponibilidade de fundos de forma sustentável; & Não \\
g) Ter a legislação concernente claramente definida; & Sim \\
h) Ter claramente definidos os mecanismos de referência e os protocolos de telemedicina; & Sim / Não \\
i) Ter recursos humanos adequados e capacitados; & Sim / Não \\
j) Garantir comunicação e articulação entre as partes interessadas. &
\end{tabular}


Tabela 4 - Os 18 fatores críticos de sucesso na implementação da telemedicina e sua observância em Cabo Verde. Adaptado de Lange M. The secret of telehealth. Ehtel: Momentum; 2014. ${ }^{24}$

\begin{tabular}{ll}
\hline Fatores ligados à estratégia e gestão & Observância em Cabo Verde \\
\hline 1. Ambiente cultural para serviços de telemedicina. & Sim \\
2. Existência de liderança através de um 'campeão', responsável pelo serviço. & Sim \\
3. Existência de consenso sobre a vantagem da telemedicina para enfrentar o 'problema' & Sim \\
identificado. & Sim \\
4. Mobilização de recursos necessários para a implementação. & Observância em Cabo Verde \\
\hline Fatores ligados à organização e gestão & Sim \\
\hline 5. Atender às necessidades do cliente primário. & Sim \\
6. Envolver os profissionais de saúde e decisores. & Não \\
7. Elaborar e implementar um plano de negócios. & Não \\
8. Elaborar e implementar um plano de gestão de mudanças. & Sim \\
9. Colocar o paciente no centro do serviço. & Observância em Cabo Verde \\
\hline Fatores ligados aos processos legais, regulamentares e de segurança & Não \\
\hline 10. Avaliar as condiçães de legalidade do serviço. & Não \\
11. Identificar e aplicar as diretrizes legais e de segurança relevantes. & Não \\
12. Envolver especialistas de leis e de segurança. & Sim \\
13. Assegurar que os prestadores de telemedicina e os utentes são 'conscientes de privacidade'. & Observância em Cabo Verde \\
\hline Fatores ligados às decisões sobre tecnologia e aquisições & Sim \\
\hline 14. Garantir que as infraestruturas de TI e de saúde em linha necessárias estão disponíveis. & Sim \\
15. Certifique-se de que a tecnologia é fácil de usar. & Sim \\
16. Instale a tecnologia e os processos necessários para monitorizar o serviço. & Sim \\
17. Mantenha um bom processo de aquisição. & Sim \\
18. Assegure-se de que a tecnologia tem o potencial para aumento de escala. &
\end{tabular}

sucesso, ligados à estratégia de implantação, à mudança organizacional, aos processos legais, regulamentares e de segurança, e às decisões sobre tecnologia e compras (Tabelas 3 e 4 ).

De destacar os seguintes pontos em comum, entre os factores críticos de sucesso mencionados por Kodukula e Nazvia e por Lange ${ }^{23,24}$ :

- A mobilização de recursos necessários para a implantação da telemedicina;

- O envolvimento de profissionais de saúde e decisores no processo de Implantação;

- Os fatores ligados aos processos legais;

- A garantia da disponibilidade de infraestruturas necessárias.

Desses factores críticos apontados por Lange, ${ }^{24}$ praticamente, todos são observados em Cabo Verde, com a exceção dos ligados à implementação de um plano de negócio, de um plano de gestão de mudanças e aos processos legais, regulamentares e de segurança, que apresentam deficiências ou, ainda, não foram tratados.

Por outro lado, a análise das entrevistas e do conteúdo dos grupos focais, permite destacar os seguintes aspectos no processo de desenvolvimento dos serviços de telemedicina, em termos de forças, fraquezas, oportunidades e ameaças:

Lipson e Henderson ${ }^{25}$ mencionam várias medidas de política para apoiar o desenvolvimento da telemedicina nos EUA, que incluem o planeamento e a coordenação, o desenvolvimento de redes, o desenvolvimento de programas específicos, o financiamento, a construção de uma infraestrutura de telecomunicações e o suporte regulatório e esclarecimentos. Porém, com frequência se ignoram as interdependências entre tecnologia, características humanas e ambiente socioeconómico, resultando em intervenções com um baixo impacto nas práticas de saúde. ${ }^{26}$

Como nos lembram Mair et al, ${ }^{27}$ além das questões organizacionais, as questões sociais devem ser consideradas, no processo de implantação da telemedicina. Igualmente, as perceções dos profissionais de saúde, as estruturas organizacionais e culturais afetando a saúde, as questões jurídicas, dificuldades técnicas, tempo, conveniência, custo, formação e familiaridade com o equipamento são elementos facilitadores para a adoção da telemedicina. ${ }^{28}$ Podem ainda ser utilizadas ferramentas de process mining para acompanhar estes processos e intervir para corrigir a implementação. ${ }^{29}$

Zanaboni e Lettieri $^{30}$ chamam a atenção para o facto dos profissionais de saúde serem, frequentemente, avessos ou indiferentes para aplicações de telemedicina. Pois, se a relação custo-eficácia pode ser considerada uma vantagem para a sociedade, para o utilizador individual esta pode não acontecer. ${ }^{22}$

\section{CONCLUSÃO}

Apesar dos avanços verificados na implantação da telemedicina em Cabo Verde, alguns factores de sucesso para a implantação da telemedicina, apontados na literatura, não se observam e, igualmente, as várias fraquezas, pontos fortes e oportunidades constatados pelos participantes no estudo têm influenciado o desenvolvimento desta intervenção no país.

\section{PROTECÇÃO DE PESSOAS E ANIMAIS}

O protocolo da pesquisa estudo foi submetido ao 
Comité de Ética Nacional que o aprovou. Os autores declaram ainda que os procedimentos seguidos estavam de acordo com a Declaração de Helsínquia da Associação Médica Mundial.

\section{CONFIDENCIALIDADE DOS DADOS}

Os autores declaram ter seguido os protocolos do seu centro de trabalho acerca da publicação de dados.

\section{REFERÊNCIAS}

1. World Health Organization. A health telematics policy in support of WHO's Health-For-All strategy for global health development: report of the WHO group consultation on health telematics, 11-16 December, 1997. Geneva: WHO; 1998.

2. Álvares S. Telemedicina: avaliação económica. NASCER E CRESCER - Rev Hospital de Crianças Maria Pia. 2004;2.

3. Lapão, LV. Lean na gestão da saúde: uma oportunidade para fomentar a centralidade do doente, o respeito pelos profissionais e a qualidade nos serviços de saúde. Acta Med Port. 2016;29:237-9.

4. Armstrong AW, Dorer DJ, Lugn NE, Kvedar JC. Economic evaluation of interactive teledermatology compared with conventional care. Telemed J E Health. 2007;13:91-9.

5. Bergmo TS. Can economic evaluation in telemedicine be trusted? A systematic review of the literature. Cost Eff Resour Alloc. 2009;24:7-18.

6. Ministério de Saúde. Relatório Estatístico de 2013. Praia: DGPOG; 2014.

7. Correia A. As evacuações médicas internas em Cabo Verde. Praia: Ministério da Saúde; 2013.

8. Ministério da Saúde. Relatório Anual do Programa Nacional de Telemedicina de 2014. Programa Nacional de Telemedicina. Praia: Ministério da Saúde; 2015.

9. Lapão LV, Correia A. Improving access to pediatric cardiology in Cape Verde via a collaborative international telemedicine service. Stud Health Technol Inform. 2015;51-7.

10. Lopes M. Potencial da telemedicina em Cabo Verde: evacuações sanitárias. Tese de Mestrado em Saúde e Desenvolvimento. Lisboa: IHMT; 2012.

11. Ministério da Saúde. Hospital Agostinho Neto. Relatório Anual. Praia: Ministério da Saúde; 2010.

12. Ministério da Saúde. Hospital Baptista de Sousa. Relatório Anual. Praia: Ministério da Saúde; 2012.

13. Ministério da Saúde. Hospital Agostinho Neto. Relatório Anual. Praia: Ministério da Saúde; 2011.

14. Ministério da Saúde. Relatório Anual do Programa Nacional de Telemedicina de 2013. Programa Nacional de Telemedicina. Praia: Ministério da Saúde; 2014

15. Ministério da Saúde. Despacho da Ministra Adjunta e da Saúde, designando os membros dos Núcleos de Telemedicina, de 25 de Março de 2013. Praia: Ministério da Saúde; 2013.

16. Ministério da Saúde. Despacho n², da Ministra Adjunta e da Saúde, de

\section{CONFLITOS DE INTERESSE}

Os autores declaram não terem qualquer conflito de interesse relativamente ao presente artigo.

\section{FONTES DE FINANCIAMENTO}

Os autores declaram não ter recebido subsídios ou bolsas para a elaboração do artigo.

17 de Janeiro de 2014, criando o Programa Nacional de Telemedicina e definindo os eixos estratégicos e os objectivos do Programa. Praia: Ministério da Saúde; 2014.

17. Ministério da Saúde. Despacho $n^{\circ} 3$, de 31 de Janeiro de 2014 nomeando o coordenador do Programa Nacional de Telemedicina. Praia: Ministério da Saúde; 2014.

18. Programa Nacional de Telemedicina. Procedimento Base de Operação. Programa Nacional de Telemedicina. Praia: PNT; 2014.

19. Programa Nacional de Telemedicina. Relatório Anual de 2013. Programa Nacional de Telemedicina. Praia: PNT; 2015.

20. Programa Nacional de Telemedicina. Relatório Anual de 2013. Programa Nacional de Telemedicina. Praia: PNT; 2014.

21. Cáceres-Méndez EA, Castro-Díaz SM, Gómez-Restrepo C, Puyana JC. Telemedicina: historia, aplicaciones y nuevas herramientas en el aprendizaje. Univ Med Bogotá. 2011;52:11-35.

22. Zanaboni $P$, Wootton R. Adoption of telemedicine: from pilot stage to routine delivery. BMC Med Inform Decis Mak. 2012;12:1.

23. Kodukula S, Nazvia M. Evaluation of critical success factors for telemedicine implementation. Int J Computer Applications. 2011;12:2936.

24. Lange M. The secret of telehealth. Ehtel: Momentum; 2014.

25. Lipson LR, Henderson TM. State initiatives to promote telemedicine. Telemed J. 1996;2:109-21.

26. van Gemert-Pijnen JE, Nijland N, van Limburg M, Ossebaard HC, Kelders SM, Eysenbach G, et al. A holistic framework to improve the uptake and impact of eHealth technologies. J Med Internet Res. 2011;13:e111.

27. Mair FS, May C, O'Donnell C, Finch T, Sullivan F, Murray E. Factors that promote or inhibit the implementation of e-health systems: an explanatory systematic review. Bull World Health Organ. 2012;90:35764.

28. Walker J, Whetton S. The diffusion of innovation: factors influencing the uptake of telehealth. J Telemed Telecare. 2002;8:S73-5.

29. Cruz-Correia R, Boldt I, Lapão L, Santos-Pereira C, Rodrigues PP, Ferreira AM, et al. Analysis of the quality of hospital information systems audit trails. BMC Med Inform Decis Mak. 2013;13:1.

30. Zanaboni $P$, Lettieri E. Institutionalizing telemedicine applications: the challenge of legitimizing decision-making. J Med Internet Res. 2011;13:e72. 